\title{
Translation, cultural adaptation and construct validity of the German version of the Adult Social Care Outcomes Toolkit for informal Carers (German ASCOT-Carer)
}

\author{
Birgit Trukeschitz ${ }^{1}$ - Assma Hajji ${ }^{1}$ Judith Litschauer ${ }^{1}$. Juliette Malley ${ }^{2} \cdot$ Adiam Schoch $^{1}$ - Stacey Rand ${ }^{3}$. \\ Ismo Linnosmaa ${ }^{4,5}$. Julien Forder ${ }^{3}$
}

Accepted: 13 October 2020 / Published online: 2 November 2020

(c) The Author(s) 2020

\begin{abstract}
Purpose The Adult Social Care Outcomes Toolkit for Carers (ASCOT-Carer), developed in England, measures the effects of long-term care (LTC) services and carer support on informal carers' quality of life (QoL). Translations of the ASCOT-Carer into other languages are useful for national and cross-national studies. The aim of this paper was to report on the translation and cultural adaptation of the original English ASCOT-Carer into German, to assess its content validity and to test for its construct validity (convergent and discriminative/known-group validity).

Methods Translation and cultural adaptation followed the ISPOR TCA guidelines. As part of the translation and adaptation process, five cognitive debriefing interviews with informal carers were used for evaluating linguistic and content validity. In addition, a sample of 344 informal carers of older adults, who received home care services in Austria, was used for hypothesis testing as suggested by the COSMIN checklist to assess convergent and discriminative/known-group validity as part of construct validity.

Results Cognitive interviews provided evidence that questions and response options of the German ASCOT-Carer were understood as intended. Associations between ASCOT-Carer scores/domains and related outcome measures (convergent validity) and expected groups of informal carers and the care service users they care for (discriminative validity) supported construct validity of the translated instrument.

Conclusion The German ASCOT-Carer instrument meets the required standards for content and construct validity which supports its usefulness for (cross-)national studies on LTC-service-related QoL-outcomes in informal carers. Research is encouraged to assess further measurement properties of the translated instrument.
\end{abstract}

Keywords Long-term care $\cdot$ Informal care $\cdot$ ASCOT $\cdot$ Cross-cultural adaptation $\cdot$ Validity

Electronic supplementary material The online version of this article (https://doi.org/10.1007/s11136-020-02682-4) contains supplementary material, which is available to authorized users.

Birgit Trukeschitz

birgit.trukeschitz@wu.ac.at

1 WU Vienna University of Economics and Business, Vienna, Austria

2 London School of Economics and Political Science, London, UK

3 University of Kent, Canterbury, UK

4 Finnish Institute for Health and Welfare, Helsinki, Finland

5 University of Eastern Finland, Kuopio, Finland

\section{Background}

Policymakers have recognized the crucial role of informal carers in supporting older and frail family members, friends, and neighbors and have since implemented benefits to improve their status and well-being [1]. To assess the carers' situation, a range of instruments [2,3] has been developed, such as the Carer Experience Scale (CES) [4, 5], the Adult Carers Quality of Life questionnaire (AC-QoL) [6], and the Adult Social Care Outcomes Toolkit for Carers (ASCOTCarer), all addressing carers' quality of life, the Zarit Burden Interview [7, 8], addressing care burden. In comparison to other tools assessing carers' quality of life (QoL), ASCOTCarer, however, is the only one to have a corresponding instrument for measuring the QoL of long-term care service 
Table 1 ASCOT-Carer domains. Source [3]

\begin{tabular}{|c|c|}
\hline Domain & Definition \\
\hline 1. Occupation & $\begin{array}{l}\text { Being sufficiently occupied in a range of meaningful, enjoyable activities whether it be formal employ- } \\
\text { ment, unpaid work, caring for others or leisure activities }\end{array}$ \\
\hline 2. Control over daily life & Choosing what to do and when to do it, and having control over their daily life and activities \\
\hline 3. Self-care (Looking after yourself) & Feeling able to look after oneself, in terms of eating well and getting enough sleep \\
\hline 4. Personal safety & $\begin{array}{l}\text { Feeling safe and secure, with concerns about safety can include fear of abuse or other physical harm or } \\
\text { accidents, which may arise as a result of caring }\end{array}$ \\
\hline $\begin{array}{l}\text { 5. Social participation and involve- } \\
\text { ment }\end{array}$ & $\begin{array}{l}\text { Being content with their social situation, whereby social situation includes the sustenance of meaningful } \\
\text { relationships with friends and family, as well as feeling involved and part of their community }\end{array}$ \\
\hline 6. Space and time to be yourself & $\begin{array}{l}\text { Having space and time in everyday life. Enough time away from caring to have a life of their own outside } \\
\text { of the caring role }\end{array}$ \\
\hline Hedra & Feeling encouraged and supported by professionals, care workers, and others, in their role as a carer \\
\hline
\end{tabular}

users [2]. Reflecting both the carers' and service users' situations makes these instruments attractive for a comprehensive assessment of long-term care (LTC) from a QoL perspective.

ASCOT-Carer was developed in England and aims to measure QoL-outcomes of (care) service provision in informal carers [2]. The instrument consists of seven distinct domains to capture areas of adult informal carers' (family and non-kin unpaid carers) everyday life that may be affected by LTC services and/or services for informal carers [3, 9]. The domains address Occupation, Control over daily life, Social participation and involvement, Selfcare (Looking after yourself), Personal safety, Feeling supported and encouraged, and Space and time to be yourself (see Table 1). Taken together, these domains form a concept known as "long-term care related quality of life (LTCQoL)", also commonly referred to as "social care related quality of life (SCRQoL)" in the English original [9]. A survey instrument that captures the effects of LTC service provision on the caregivers' QoL contributes to improving empirical evidence and thus to a better understanding of how LTC services meet the needs of informal carers in different aspects of their lives.

The ASCOT-Carer instruments consist of an interview version with four response levels (INT4) and a self-completion tool with four response levels (SCT4) ${ }^{1}$ which reflect different outcome states: Ideal state (3)_individual's preferences are met, No needs (2)_individual's needs are met but not to the desired level, Some needs (1)-there are unmet needs, but no health implications, High-level needs (0)_ needs have an immediate or longer-term health implication $[2,9]$. The total ASCOT-Carer score is the total raw score of the seven domains and ranges from 0 (worst state) to 21 (ideal state). As each domain represents a distinct aspect of the latent construct SCRQoL, weighted combinations of

$\overline{1} \mathrm{https} / / / \mathrm{www} . p s s r u . a c . u k / a s c o t /$ tools/. indicators are recommended (Avila et al. 2015). ASCOTCarer was developed as a preference-weighted measure [9] to reflect the value of the care service-induced gain in QoL.

The ASCOT-Carer instrument has gained considerable interest in non-English-speaking countries and has been translated into Japanese, Dutch and Finnish. ${ }^{2}$ The availability of instruments in different languages makes it possible to investigate QoL-effects of LTC service provision on informal carers across nations and within a country among speakers of different languages. A culturally valid adaptation of the ASCOT-Carer instrument can contribute to strengthening the evidence base for policy decisions on how to improve LTC-service provision and the living situation of informal carers. For the translated instrument to be used with confidence, there needs to be cross-cultural equivalence between the valid translated and the original questionnaires [10].

Methodological approaches aiming to establish and evaluate cross-cultural equivalence in questionnaires address both the translation and cultural adaptation process and the assessment of measurement properties of the translated instrument. Translation and cultural adaptation (together also referred to as "cross-cultural adaptation") seek to improve equivalence on a semantic, idiomatic, experiential and conceptual level [11]. For this purpose, systematic multistep guidelines and qualification recommendations for key actors involved in this process have been established (e.g. [11-13]). The assessment of the measurement model of the construct is essential as it provides the starting point for the choice of methods to evaluate the measurement properties of the culturally adapted instrument. Reflective and formative measurement models differ in terms of the relationship between the construct and its indicators [14] with respect to the nature of the construct, the direction of causality and the characteristics of indicators [15]. In formative models, to which the ASCOT-Carer instruments

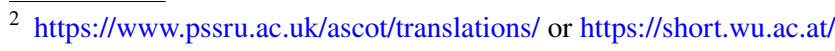
ascot.
} 
conceptually belong to, a latent construct (here SCRQoL) is formed by its items (seven ASCOT-Carer domains); variation in the latent construct of SCRQoL is caused by variation in the ASCOT-Carer domains. These seven domains define the construct and are thus not interchangeable, nor do they share a singular common theme. Adding or dropping an ASCOT-Carer domain may change the conceptual meaning of the SCRQoL-construct. In formative measurement models, content and construct validity thus play an important role for assessing validity, defined as the extent to which the interpretation of the results of the measure are warranted [16], at the score and item level. Methods to assess structural validity, such as confirmative factor analysis, and internal consistency, measured by Cronbach's alpha, are appropriate for reflective, but not for formative models, in which items may correlate positively, negatively or not at all [14].

The aim of this study was to translate and culturally adapt the original English-language ASCOT-Carer instruments (INT4 and SCT4) into German and to examine aspects of validity of the translated instrument. We report on the results of assessing linguistic and content validity as part of the translation and adaptation process. In addition, we investigate construct validity of the translated measure using survey data for testing expected relationships of the German ASCOT-Carer instrument with comparator outcome measures (convergent validity) and with selected subgroup characteristics of informal carers (discriminative or known-groups validity). As the ASCOT-Carer instrument follows a formative model, each domain is relevant for defining the latent construct. Thus, we assess construct validity on both the score and item level of the translated instrument. We shed light on challenges for cultural adaption and validation and discuss solutions to these challenges that may inform future studies on adapting the English ASCOT-Carer instrument into other languages. A valid German version of the ASCOT-Carer instruments will be a useful tool for national and cross-national surveys on the effects of LTC services on the QoL of German speaking informal carers.

The rest of the paper is organized as follows: First, the methods section describes the approaches applied for cultural adaption, the methods for assessing linguistic, content and construct validity. Then, results of the current study are presented, followed by a discussion of the main findings and comparison to previous work on validation of the original English ASCOT-Carer.

\section{Methods}

\section{ASCOT-Carer translation into German and cultural adaption}

The ASCOT-Carer instruments (INT4, SCT4) were translated from English into German between June 2015 and
March 2016 by the Austrian research team in cooperation with the translation agency PharmaQuest (now part of Corporate Translations, Inc.) and the English ASCOT development team. In line with ISPOR's principles of good practice for the translation and cultural adaptation (TCA) process for patient-reported outcome (PRO) measures [12], key actors and methods were chosen to ensure appropriate cross-cultural equivalence of the English and German ASCOT-Carer. The translation company involved four bilingual translators, an in-country investigator familiar with the country's care practice, and one independent proofreader. The Austrian research team had considerable experience with ASCOT as well as with care policy and practice in German speaking countries. The ASCOT development team was previously involved in the cultural adaption of ASCOT (service user measure) into other languages.

Figure 1 describes the steps of the translation and crosscultural adaptation process [17] following Wild et al. [11]. The ASCOT-Carer concept clarification guide, previously produced by the translation company and approved by ASCOT development team, defined the conceptual meaning of each item and its response options of the ASCOTCarer measures (Step 1). Based on this guidance, translators, native in German and fluent in English, drafted two initial translations (Step 2). These were then reconciled into a single version by an in-country investigator who held a degree in translation and interpreting and had worked as a nurse (Step 3). The revised version was back-translated into English independently by two translators, native in English and fluent in German (Step 4) for further review by the translation agency's in-country investigator, the ASCOT development team and the German-speaking research team (Step 5). Step 6 of the ISPOR TCA guideline addresses the process of harmonization which aims to consider all new translations (into different languages). As at the time of translation German and Finnish versions were the first ASCOT-Carer instruments to be translated, the Austrian and Finnish teams shared and discussed their results at key steps 5 and 7 of the adaptation process. Going beyond the ISPOR TCA guidelines, the revised German version was then proofread by an independent translator, not previously involved in the translation process, and reviewed by the Austrian research team, who also conducted professional reviews with an Austrian care worker and a care manager. Then, cognitive debriefings with five informal carers were carried out by the Austrian research team (Step 7) and comments were sent to the translation agency. The translation agency reviewed the results of the cognitive debriefing (Step 8) and made amendments where necessary. The pre-final version was proofread (Step 9) by the in-country investigator before being approved by the translation agency (Step 10). The original English version and the final German translation of the ASCOT-Carer 


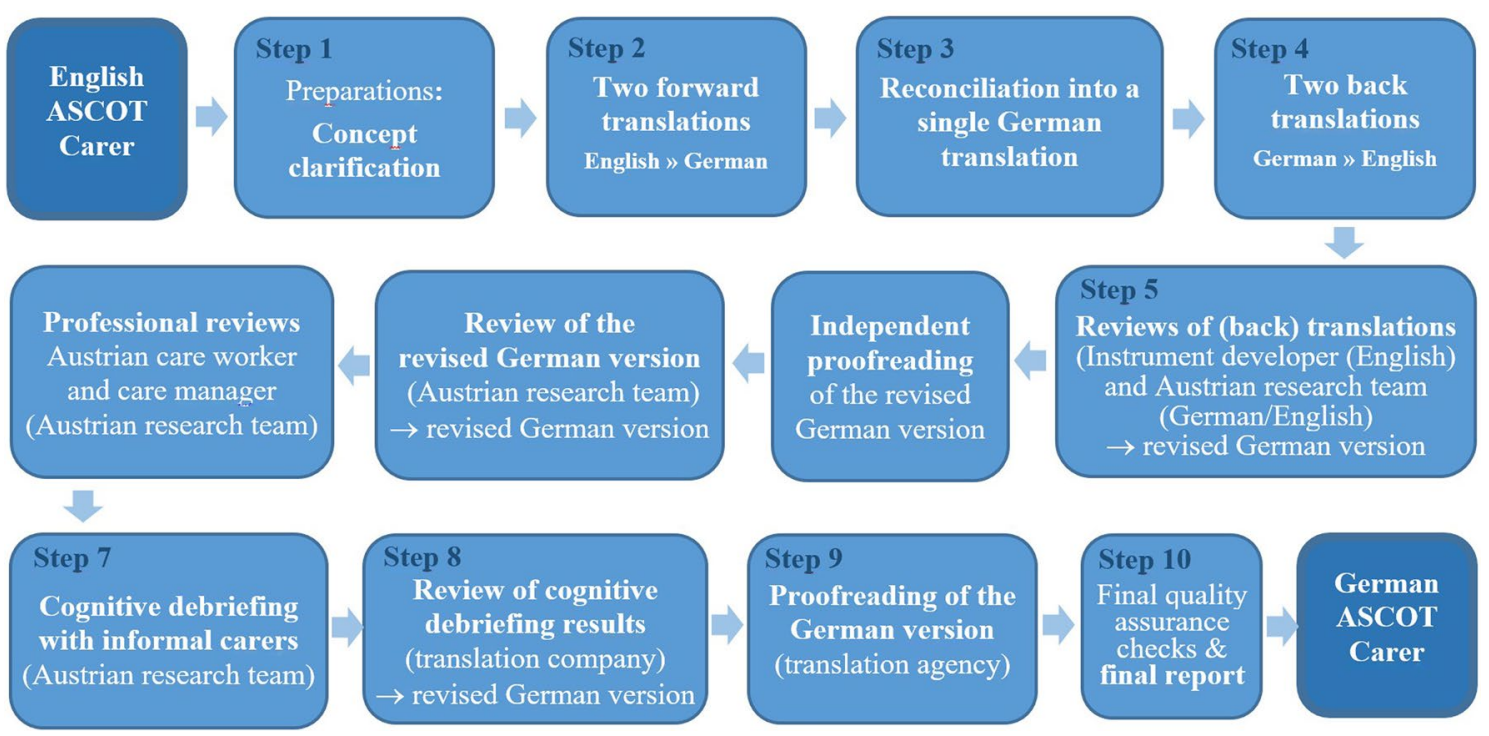

Fig. 1 Flow diagram of the translation and cross-cultural adaptation process of ASCOT-Carer (English into German) following ISPOR TCA. Notes: Step 6-harmonization of new translations (in our case

instruments (INT4 and SCT4) are available on the ASCOT website (www.pssru.ac.uk/ascot or https://short.wu.ac.at/ ascot).

\section{Data collection}

We used two data sources to improve cultural adaptation and to assess the construct validity of the German version of the ASCOT interview for informal carers (ASCOT-Carer INT4): Cognitive debriefings as part of the translation and adaptation process aimed to give insight into the understanding of question and response option intent and the meaning of terms to the respondents. Two researchers (one woman, one man), trained in conducting cognitive interviews with older people, were provided with a comprehensive interview guide that comprised general instructions for the interviewers as well as tasks and questions for the interviewees right after each ASCOT-Carer item and the related set of response options. All cognitive debriefing interviews were recorded. After each interview, experiences and first results were reflected on in the Austrian research team. As per the ISPOR's TCA group [11] and the translation agency's recommendations [17], five face-to-face cognitive interviews were carried out in November and December 2015, with the possibility of increasing the sample size if needed. As the initial analysis of the transcripts showed a great deal of overlap in how the respondents understood the items and little variation in terms of the types of misunderstandings, no further interviews were carried out. The respondents were informal carers whose relatives received home care services from care service providers in Vienna. Each respondent was with the Finnish translation)—was part of key steps 5 and 7. Source: PharmaQuest Ltd. [17], authors' illustration

asked to fill out a copy of the whole translated questionnaire, to comment on the wording of questions and response options that were difficult to understand, and to suggest alternative wordings throughout the process. The cognitive debriefings with informal carers combined the think-aloud method with verbal probing techniques [18].

In addition, cross-sectional quantitative data on informal carers of home care service users were collected via a survey in 2016/17 as part of the EXCELC project in Austria. In a first step, home care service users in all nine Austrian provinces were recruited for study participation in cooperation with the Federal Ministry of Labor, Social Affairs and Consumer Protection as well as local authorities and care organizations. Service users who reported receiving informal help were asked to provide contact information for their main informal carer, who was then interviewed face-to-face using a standardized questionnaire. In total, 344 informal carers of LTC service users were interviewed across all nine Austrian regions (Laender) using computer-aided personal standardized interviews (CAPI) designed by the online survey software 'Qualtrics'. ${ }^{3}$ The interviewers used showcards for the ASCOT-Carer section in the questionnaire. Details on the data collection are reported elsewhere [19].

\footnotetext{
3 https://www.qualtrics.com/de/.
} 


\section{Assessing linguistic and content validity of the German ASCOT-Carer}

Cognitive interview transcripts were used to assess linguistic and content validity to inform changes needed to further improve the cross-cultural adaptation of the translated ASCOT-Carer. Linguistic validity ensures the conceptual and linguistic equivalence between the original and translated version of the instrument [20]. An item-based analysis was performed in order to identify discrepancies in meaning between the original and translated versions of ASCOTCarer items or response options. It consisted of summarizing responses, identifying problem areas and proposing suggestions and amendments where needed [21]. Content validity refers to how well an instrument's content reflects the constructs to be measured [22] and whether it is comprehensive, understandable and acceptable [23]. Cognitive interview transcripts and the ASCOT concept clarification guide $[9,12]$ were used to assess whether the translation adequately reflected the original content of the ASCOT items and response choices.

\section{Assessing construct validity of the German ASCOT-Carer}

Survey data were taken to assess the construct validity of the German translation of the ASCOT-Carer instrument. The COSMIN (COnsensus-based Standards for the selection of health Measurement INstruments) checklist [24] recommends hypotheses testing for investigating construct validity (box 9a+b) of the translated instrument [24, 25]. We performed a series of bivariate tests of association to examine expected relationships between the ASCOT-Carer measure with comparator outcome measurement instruments to assess convergent validity and with selected subgroups of informal carers to evaluate discriminative or known-groups validity. A complete overview of the measures expected to be related with the German version of the ASCOT-Carer instrument is given in Table 8 in the Online Appendix.

Based on theoretical considerations or previous empirical evidence, we expected the overall ASCOT-Carer score to be related with scores of comparator outcome measures, such as QoL and health-related QoL as well as indicators of care experience and care burden [3]. The variables used for testing the hypothesized relationships include self-perceived quality of life (QoL) as a seven point measure (seven indicating the highest possible QoL), and health-related QoL, measured by EQ-5D-3L items (high values indicating better states) and the EQ-5D index (anchored at 0 for death and 1 for perfect health using German weights [26, 27]). As indicators of care burden, we used the Carer Experience Scale (CES) [4] (ranging from 0 to 18 , with high values indicating better states), the Zarit Burden Interview (ZBI) using the four-item screening version [28] (ranging from 0 to 14 , with high values indicating high caregiving burden), questions on service users' cognitive performance and behavior and care intensity measured in hours. To evaluate the validity of the individual ASCOT-Carer domains, we used items of the outcome measures that are expected to reflect the individual domains of the translated measure. For the comparison between subgroups of informal carers, we compared informal carers with high and low care intensity (measured by care hours per week), carers' opportunity of getting break from caring or not and informal carers who cared for relatives or non-kin with/without cognitive skills or challenging behavior. We also used selected items of comparator outcome measures (selected item of EQ-5D and ZBI) to form groups of carers to investigate the relationships with selected ASCOT-Carer domains.

For ASCOT-Carer scores related to comparative outcome scores, we used Spearman's rho to assess correlations. Thresholds for association effect sizes were chosen according to Cohen [29]. Fisher's exact tests (for categorical variables) and one-way analyses of variance, ANOVA, (for continuous variables) were used to test the hypothesized associations for ASCOT-Carer domains. Benjamini and Hochberg [30] correction for multiple testing was applied. For ANOVA results, omega squared was chosen to assess effect size as it is less biased then eta squared in smaller samples [31]. For the thresholds see Cohen [29]. Convergent validity is perceived as adequate if more than $75 \%$ of the expected relationships can be supported by the analysis results or if a correlation between the measures expected to be related is stronger than 0.5 [32]. All statistical analyses were conducted in Stata v15 [33].

\section{Results part 1}

\section{Cultural adaptation during the translation process}

Some sections of the translation of the ASCOT-Carer instruments into German required cultural adaptation to appropriately reflect the meaning for the German-speaking target group. First, a few English expressions challenged the forward translation into German (e.g. "control over daily life", "to feel clean and presentable", "thinking about myself", "to be yourself in your daily life") as there was no meaningful direct translation. Where no appropriate final wording was found in the translation process, alternative expressions were tested in the professional review and cognitive debriefing phases (e.g. for "control over daily life"). Second, we found that some translations into German resulted into back translations that did not literally match the original because of idiomatic expressions which were preferred to literal translations (e.g. 'role' as a carer was changed to 'task' of a carer). 
Third, contemporary German language seeks to account for the gender of the actors, e.g. 'der Interviewer' (male interviewer), 'die Interviewerin' (female interviewer). As English usually has no grammatical gender, the gender of German nouns (masculine, feminine or neutral) added complexity to sentences referring to actors. Last but not least, cultural adaptation was needed for the terms referring to specific actors (e.g. carers), services and institutions (e.g. the British National Health Service) in the English and Austrian longterm care systems.

\section{Linguistic and content validity of the German ASCOT-Carer}

Linguistic and content validity were also evaluated by cognitive debriefing interviews. Five female informal carers, aged 46-72, caring for their relatives between one and 20 years, were involved in the cognitive debriefing to assess how well questions and response options were understood and whether the wording was appropriate.

The cognitive interviews showed that the respondents' comprehension of the German translation of the ASCOTCarer instrument was satisfactory, as they were able to adequately explain their responses with respect to each of the ASCOT-Carer domains. Minor changes to the wording were made in cases where at least one interviewee expressed confusion or ambiguity or misunderstood a part of the item (e.g. the preferred translation for 'support' was 'Unterstützung' (assistance), not 'Hilfe' (help)). These changes to the translation were made in accordance with the English ASCOT development team. Respondents generally understood the four response options as intended and were able to distinguish between them without difficulties. We used different German expressions ('wie ich es will/möchte/mir vorstelle', i.e. 'the way I want/would like') for the ideal state phrased 'as I want' in the original English tool as they seemed more suited to the respective specific contexts.

The Control over daily life domain could not easily be translated over. Thus, two options for an adaptation were tested with informal carers. The cognitive interviews for this domain revealed that option 1 'nach eigenem Ermessen' (at their own discretion) was perceived as too broad a concept whereas option 2 'selbstbestimmt gestalten' (being able to influence) was viewed as more specific and tangible. The second option was better understood and, therefore, used in the final questionnaire for the quantitative data collection.

The domains Self-care, Social participation and involvement, and Occupation were understood as intended. As interviewees preferred short names for ASCOT-Carer domains, the final wording for the Social participation and involvement domain was 'Sozialleben' (social life) instead of 'Soziale Kontakte und Engagement im gesellschaftlichen Lebens' (social contacts and social involvement). One of the response options for the Occupation domain was changed to 'Ich verbringe etwas, aber nicht ausreichend Zeit' instead of 'Ich verbringe einige, aber nicht ausreichend Zeit' ('I do some of the things I value or enjoy with my time, but not enough'), as the German term 'einige' was understood to mean 'a lot of'. The German translation of the questions about Feeling supported and encouraged was associated, as intended, with the feeling of appreciation and empathy from others, but also with financial support. Some respondents thought about having support in general (the fact that a care worker comes) while others reflected more on the actual support they were receiving by particular care workers or services. Informal carers, who were not in direct contact with care workers, seem to be less likely to feel supported by them and tended to think about support in more general terms. For one respondent, the idea of feeling supported as an informal carer did not seem relevant (this might be due to the fact that the respondent was also employed in the care sector and did not feel the need to rely on getting support).

Personal safety turned out not always to be understood in the same way. Some respondents did not find it easy to define or delimit the concept of personal safety and included financial security. In addition, some reflected on safety in connection with formal help. Feelings of better safety seemed to result from receiving regular formal help and from having trust in the care workers. On the other hand, some characteristics of service provision, such as frequent changes of care workers, seemed to negatively affect feelings of safety. While some respondents refer to injury risk and physical strain due to caregiving, others had difficulties to understand how safety is related to their caregiving and had a different idea of safety in mind than being safe from accidents and abuse. These different ways to interpret 'personal safety' stress the importance of using the interviewer prompt with this domain ('with 'feeling safe' we mean feeling safe from fear of abuse, being attacked or other physical harm, such as accidents, which are a result of your caring role').

The German translation of the questions about Space and time to be yourself captured, as intended, the carers' ability to have enough time away from caring and to have a life of their own outside of the caring role. One respondent interpreted 'space' literally as having a separate apartment, not shared with the cared-for person, and thus having a life outside of the caring role.

Based on these findings, a few recommendations for amendments to the questionnaire were sent to the translation agency, which incorporated changes in consultation with the Austrian researchers and the ASCOT development team. The translated version (following proofreading and final checks) was then taken forward in the field phase (quantitative data collection). 
Table 2 Sample characteristics: informal carers (Austria). Source WU, EXCELC INT C AUT 2016/2017, $n=344$

\begin{tabular}{|c|c|c|}
\hline & Frequency & $\%$ \\
\hline \multicolumn{3}{|l|}{ Sex } \\
\hline Female & 233 & 67.7 \\
\hline Male & 111 & 32.3 \\
\hline \multicolumn{3}{|c|}{ Relationship to person cared for } \\
\hline Partner & 132 & 38.4 \\
\hline Child & 177 & 51.4 \\
\hline Other & 34 & 9.9 \\
\hline Missing & 1 & 0.3 \\
\hline \multicolumn{3}{|l|}{ Co-residence } \\
\hline Yes & 218 & 63.4 \\
\hline No & 125 & 36.3 \\
\hline Missing & 1 & 0.3 \\
\hline \multicolumn{3}{|l|}{ Duration of caring } \\
\hline Less than a year & 14 & 4.07 \\
\hline 1 year, less than 3 years & 74 & 21.51 \\
\hline 3 years, less than 5 years & 77 & 22.38 \\
\hline 5 years, less than 10 years & 90 & 26.16 \\
\hline More than 10 years & 85 & 24.71 \\
\hline Missing & 4 & 1.16 \\
\hline \multicolumn{3}{|l|}{ Informal care hours per week } \\
\hline Less than 10 hours/week & 67 & 19.5 \\
\hline 10-49 hours/week & 140 & 40.7 \\
\hline 50-99 hours/week & 55 & 16.0 \\
\hline $100+$ hours/week & 69 & 20.1 \\
\hline Missing & 13 & 3.8 \\
\hline \multicolumn{3}{|l|}{ Services for informal carers } \\
\hline Yes & 84 & 24.4 \\
\hline No & 260 & 75.6 \\
\hline Total & 344 & 100.0 \\
\hline
\end{tabular}

Percentages may not total $100.0 \%$ due to rounding

\section{Results part 2}

\section{Survey sample characteristics}

In total, 344 informal carers completed the standardized personal interviews (which meets the recommended sample size of 100 and more respondents for hypothesis testing for construct validity [13]). Table 2 shows the sample characteristics: two thirds of the informal carers in the sample were women, just over half of the sample was the child of the cared-for person and 38\% were the partner, all others were other family members and non-kin, such as friends or neighbors. Almost two thirds of the informal carer sample lived in the same household as the home care service user. About half of the informal carer sample supported their relatives or non-kin less than 5 years. Of the sample, $20 \%$ spent less than 10 hours per week in caregiving, $40 \%$ between 10 and 50 hours, 16\% 50-99 hours and 20\% more than 100 hours per week. In total, only one in four informal carers had taken up services for carers, such as information and advice, support from a carers' group or short-term care. Details concerning the study design, data collection and extensive sample characteristics of the Austrian informal carer data are provided elsewhere [19].

The distribution of responses for each ASCOT-Carer domain and the distributional statistics for the overall ASCOT-Carer score are shown in Table 3. The percentage of missing values was generally low, ranging from $0.2 \%$ (Self-care) to $3.5 \%$ (Feeling supported and encouraged), indicating a good acceptance of the German version of the ASCOT-Carer instrument. For this reason, no imputation was performed when investigating item-level and overall validity. Individuals with missing values in at least one of the domains were therefore not considered in the validation of the total score, they were, however, included in

Table 3 Responses to the German ASCOT questionnaire for informal carers (German ASCOT-Carer). Source WU, EXCELC INT C AUT 2016/2017, $n=344$

\begin{tabular}{|c|c|c|c|c|c|c|c|}
\hline $\begin{array}{l}\text { German ASCOT- } \\
\text { Carer response } \\
\text { options }\end{array}$ & Occupation Freq. (\%) & Control Freq. (\%) & $\begin{array}{l}\text { Social partici- } \\
\text { pation Freq. } \\
(\%)\end{array}$ & $\begin{array}{l}\text { Feeling sup- } \\
\text { ported Freq. } \\
(\%)\end{array}$ & Self-care Freq. $(\%)$ & $\begin{array}{l}\text { Personal } \\
\text { safety Freq. } \\
(\%)\end{array}$ & $\begin{array}{l}\text { Space } \\
\text { and time } \\
\text { Freq. (\%) }\end{array}$ \\
\hline High needs & $12(3.49)$ & $22(6.40)$ & $11(3.20)$ & $16(4.65)$ & $39(11.34)$ & $6(1.74)$ & $13(3.78)$ \\
\hline Some need & $147(42.73)$ & $106(30.81)$ & $74(21.51)$ & $73(21.22)$ & $94(27.33)$ & $28(8.14)$ & $111(32.27)$ \\
\hline No needs & $117(34.01)$ & $148(43.02)$ & $127(36.92)$ & $139(40.41)$ & $135(39.24)$ & 109 (31.69) & $159(46.22)$ \\
\hline Ideal state & $66(19.19)$ & $66(19.19)$ & $130(37.79)$ & $104(30.23)$ & $75(21.80)$ & $198(57.56)$ & $59(17.15)$ \\
\hline$\underline{\text { Missing }}$ & $2(0.58)$ & $2(0.58)$ & $2(0.58)$ & $12(3.49)$ & $1(0.23)$ & $3(0.87)$ & $2(0.58)$ \\
\hline \multicolumn{4}{|c|}{ Mean (SD) } & Median & Min & Max & $n$ \\
\hline German ASCOT- & er: index (range: $0-21$ ) & \multicolumn{2}{|c|}{13.45 (4.13) } & 14 & 2 & 21 & 328 \\
\hline
\end{tabular}


Table 4 Significance of correlations between ASCOT-Carer score and scores of comparator outcome measurement instruments. Source WU, EXCELC INT C AUT 2016/2017

\begin{tabular}{lcc}
\hline $\begin{array}{l}\text { Scales expected to be } \\
\text { related }\end{array}$ & $\begin{array}{l}\text { ASCOT-Carer score } \\
\text { Spearman's rho }(p \text {-value })\end{array}$ & $n$ \\
\hline EQ-5D index & $0.382(<0.001)$ & 324 \\
QoL $^{\text {a }}$ & $0.501(<0.001)$ & 169 \\
CES score & $0.370(<0.001)$ & 299 \\
ZBI score & $-0.541(<0.001)$ & 320 \\
\hline
\end{tabular}

Thresholds according to Cohen [29]: 0.10 for small, 0.30 for medium and 0.50 for large effect sizes

${ }^{\text {a }}$ The seven-point QoL-scale was added to the questionnaire at a later stage in the field phase, hence the lower number of respondents

domain-specific analyses for the domains in which they had given valid answers.

Item response distributions can also be used to assess potential floor or ceiling effects, which would manifest themselves in particularly high cell counts in the highest or lowest categories. Generally, frequencies were highest in the middle categories except for the domains of Social participation and involvement and Personal safety, where most respondents were in the 'ideal state' category. As all domains are scaled according to a similar logic, high levels of 'ideal state' answers in those two categories are not seen as indication of a ceiling effect, but rather a reflection of the respondents' situations in these specific areas of life.

\section{Construct validity of the German ASCOT-Carer}

\section{Construct validity of the overall ASCOT-Carer score (German version)}

To evaluate construct validity, we first examined correlations of the ASCOT-Carer total score (LTC-QoL score) with related concepts (Table 4). Significant, albeit moderate, correlations [29] were found for all variables (EQ-5D index, overall QoL, CES and ZBI as well as weekly care hours) with the overall ASCOT-Carer score. As expected, the EQ-5D index (H3), QoL (H1) and CES (H2) were positively correlated with ASCOT-Carer score, suggesting that better health-related QoL (EQ-5D), overall QoL (7-point QoL scale) and carer's experience (CES score) were associated with a higher LTC-QoL for carers. ZBI score $(\mathrm{H} 4)$ was negatively correlated with the ASCOT-Carer score which suggests, as hypothesized, that negative experiences of caregiving (high ZBI-values, indicating, for example, being stressed and unsure) relate to low LTC-QoL for informal carers.

\section{Domain-specific construct validation of the ASCOT-Carer (German version)}

Table 5 summarize the results for scores of comparator outcome measures to be related to all seven ASCOT-Carer domains, state the significance and direction of the association. As expected, a number of instruments (QoL (h1), EQ-5D index (h2), CES (h3) and ZBI (h4)) scores were related to all ASCOT-Carer items. In general, associations were significant at the $1 \%$ level (see Table 5) for CES and $\mathrm{ZBI}$ as well as EQ-5D, the latter with the exception of Occupation (10\%), Self-Care (5\%) and Space and time to be yourself (not significant).

Tables 6 and 7 shows the results for single items of comparator outcome measures to be related to ASCOT-Carer domain and specific subgroups of informal carers expected to be related with the ASCOT-Carer domains.

\section{Occupation}

As expected, the EQ-5D item 'usual activities' (h-occu1) and the CES item 'life outside caring' (h-occu2) were significantly positively, and the ZBI item 'time for oneself' (h-occu3) negatively associated with the ASCOT-Carer item Occupation, which aims to capture meaningful and enjoyable activities. In addition, associations between Occupation and characteristics of the care setting, namely challenging behavior (h-occu5) and cognitive skills of the cared-for person (h-occu6) and care hours (h-occu4) were associated in the expected direction at the 5\% (challenging behavior) or $1 \%$ level, suggesting that challenging behavior and low cognitive skills as well as many weekly care hours were related to low QoL in the domain Occupation of the German ASCOT-Carer (Tables 6 and 7).

\section{Control over daily life}

In line with the results of the validation for the English original [3], we found a significant positive association between the EQ-5D item 'usual activities' and the ASCOT-Carer item Control over daily life (h-cont1). As expected, the ZBI items 'stressed because of compatibility of caring and other responsibilities' (h-cont3) and 'feeling uncertain about service user' (h-cont4) were negatively associated with Control. The CES item 'control over caring', however, was not found to be positively associated (h-cont 2 , not confirmed). We found a highly significant negative association between the Control item and weekly care hours (h-cont5), with higher care hours per week being related with lower feelings of being in control of daily life (Tables 6 and 7). 
Table 5 EQ-5D, CES and ZBI scores by the German ASCOT-Carer domains. Source WU, EXCELC INT C AUT 2016/2017

\begin{tabular}{|c|c|c|c|c|}
\hline ASCOT-Carer attributes per domain & QoL $(n=169)$ & $\mathrm{EQ}-5 \mathrm{D}(n=338)$ & CES $(n=309)$ & $\mathrm{ZBI}(n=333)$ \\
\hline \multicolumn{5}{|l|}{ Occupation } \\
\hline High needs & $3.14(7)$ & $0.90(12)$ & $11.82(11)$ & $5.67(12)$ \\
\hline Some needs & $3.41(76)$ & $0.84(145)$ & $12.75(133)$ & $7.07(142)$ \\
\hline No needs & $4.07(55)$ & $0.89(116)$ & $14.11(108)$ & $4.89(113)$ \\
\hline Ideal state & $4.13(31)$ & $0.90(65)$ & $14.35(57)$ & $3.36(66)$ \\
\hline$F$ statistic and significance & $10.06 * * *$ & $2.47 *$ & $8.77 * * *$ & $25.03 * * *$ \\
\hline Omega squared & 0.14 & 0.01 & 0.07 & 0.18 \\
\hline \multicolumn{5}{|l|}{ Control over daily life } \\
\hline High needs & $3.27(11)$ & $0.83(22)$ & $11.32(19)$ & $7.00(21)$ \\
\hline Some needs & $3.35(54)$ & $0.83(104)$ & $13.11(96)$ & $7.12(101)$ \\
\hline No needs & $3.96(70)$ & $0.89(146)$ & $13.83(137)$ & $5.35(146)$ \\
\hline Ideal state & $4.09(34)$ & $0.92(66)$ & $14.02(57)$ & $3.08(65)$ \\
\hline$F$ statistic and significance & $8.09 * * *$ & $4.49 * * *$ & $6.19 * * *$ & $25.19 * * *$ \\
\hline Omega squared & 0.11 & 0.03 & 0.05 & 0.18 \\
\hline \multicolumn{5}{|l|}{ Social participation and involvement } \\
\hline High needs & $3.31(42)^{\mathrm{a}}$ & $0.76(11)$ & $10.55(11)$ & $8.91(11)$ \\
\hline Some needs & & $0.82(72)$ & $12.20(66)$ & $6.83(70)$ \\
\hline No needs & $3.81(70)$ & 0.89 (127) & $13.84(115)$ & $5.52(123)$ \\
\hline Ideal state & $4(56)$ & $0.89(128)$ & $14.16(117)$ & $4.56(129)$ \\
\hline$F$ statistic and significance & $5.72 * * *$ & $4.84 * * *$ & $13.33 * * *$ & $11.83 * * *$ \\
\hline Omega squared & 0.08 & 0.03 & 0.11 & 0.09 \\
\hline \multicolumn{5}{|l|}{ Feeling supported and encouraged } \\
\hline High needs & $3.71(7)$ & $0.81(15)$ & $10.27(15)$ & $7.06(16)$ \\
\hline Some needs & $3.08(37)$ & $0.81(73)$ & $11.55(64)$ & $7.53(72)$ \\
\hline No needs & $3.76(71)$ & $0.88(138)$ & $13.97(128)$ & $5.77(133)$ \\
\hline Ideal state & $4.18(51)$ & $0.91(102)$ & $14.56(96)$ & $3.74(103)$ \\
\hline$F$ statistic and significance & $12.39 * *$ & $5.15^{* * *}$ & $30.72 * * *$ & $23.92 * * *$ \\
\hline Omega squared & 0.17 & 0.04 & 0.23 & 0.18 \\
\hline \multicolumn{5}{|l|}{ Self-care (Looking after yourself) } \\
\hline High needs & $2.86(16)$ & $0.80(38)$ & $11.22(37)$ & $7.41(39)$ \\
\hline Some needs & $3.44(48)$ & $0.86(94)$ & $13.31(85)$ & $7.07(89)$ \\
\hline No needs & $3.91(68)$ & $0.88(134)$ & $13.98(120)$ & $5.13(131)$ \\
\hline Ideal state & $4.22(37)$ & $0.91(73)$ & $14.10(68)$ & $3.43(75)$ \\
\hline$F$ statistic and significance & $12.99 * * *$ & $3.4 * *$ & $11.99 * * *$ & $25.23 * * *$ \\
\hline Omega squared & 0.18 & 0.02 & 0.10 & 0.18 \\
\hline \multicolumn{5}{|l|}{ Personal safety } \\
\hline High needs & $3.19(16)^{\mathrm{a}}$ & $0.71(6)$ & $12.50(6)$ & $7.33(6)$ \\
\hline Some needs & & $0.71(28)$ & $11.85(26)$ & $7.93(28)$ \\
\hline No needs & $3.50(68)$ & $0.86(108)$ & $12.98(99)$ & $6.10(105)$ \\
\hline Ideal state & $4.05(85)$ & $0.90(195)$ & $14.03(177)$ & $4.81(193)$ \\
\hline$F$ statistic and significance & $11.32 * * *$ & $12.89 * * *$ & $7.11 * * *$ & $9.95^{* * *}$ \\
\hline Omega squared & 0.10 & 0.10 & 0.06 & 0.07 \\
\hline \multicolumn{5}{|l|}{ Space and time to be yourself } \\
\hline High needs & $3.00(6)$ & $0.80(13)$ & $10.23(13)$ & $8.77(13)$ \\
\hline Some needs & $3.43(54)$ & $0.86(109)$ & $12.93(100)$ & $7.17(106)$ \\
\hline No needs & $3.94(82)$ & $0.88(159)$ & $13.97(145)$ & $4.95(155)$ \\
\hline Ideal state & $3.96(27)$ & $0.90(57)$ & $14.04(51)$ & $3.47(59)$ \\
\hline$F$ statistic and significance & $5.72 * * *$ & $1.7 \mathrm{n} . \mathrm{s}$ & $10.39 * * *$ & $26.35 * * *$ \\
\hline Omega squared & 0.08 & & 0.08 & 0.19 \\
\hline
\end{tabular}

Thresholds according to Cohen [29]: 0.01 for small, 0.06 for medium and 0.14 for large effect sizes n.s. not significant

***Significant at $1 \%$ level, **significant at $5 \%$ level, *significant at $10 \%$ level

${ }^{a}$ Lowest two levels (high needs and some needs) were collapsed for the analysis due to low cell counts 


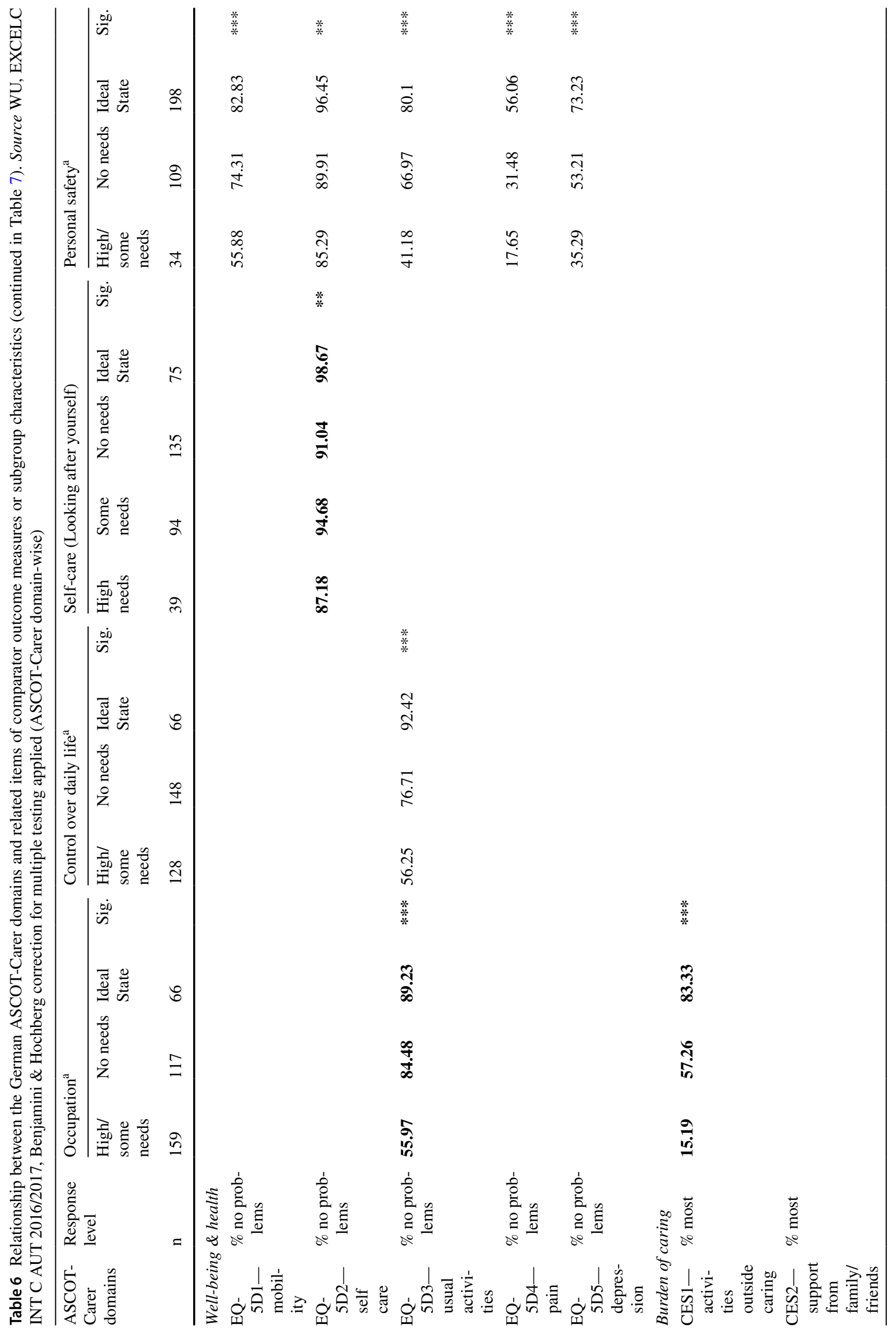




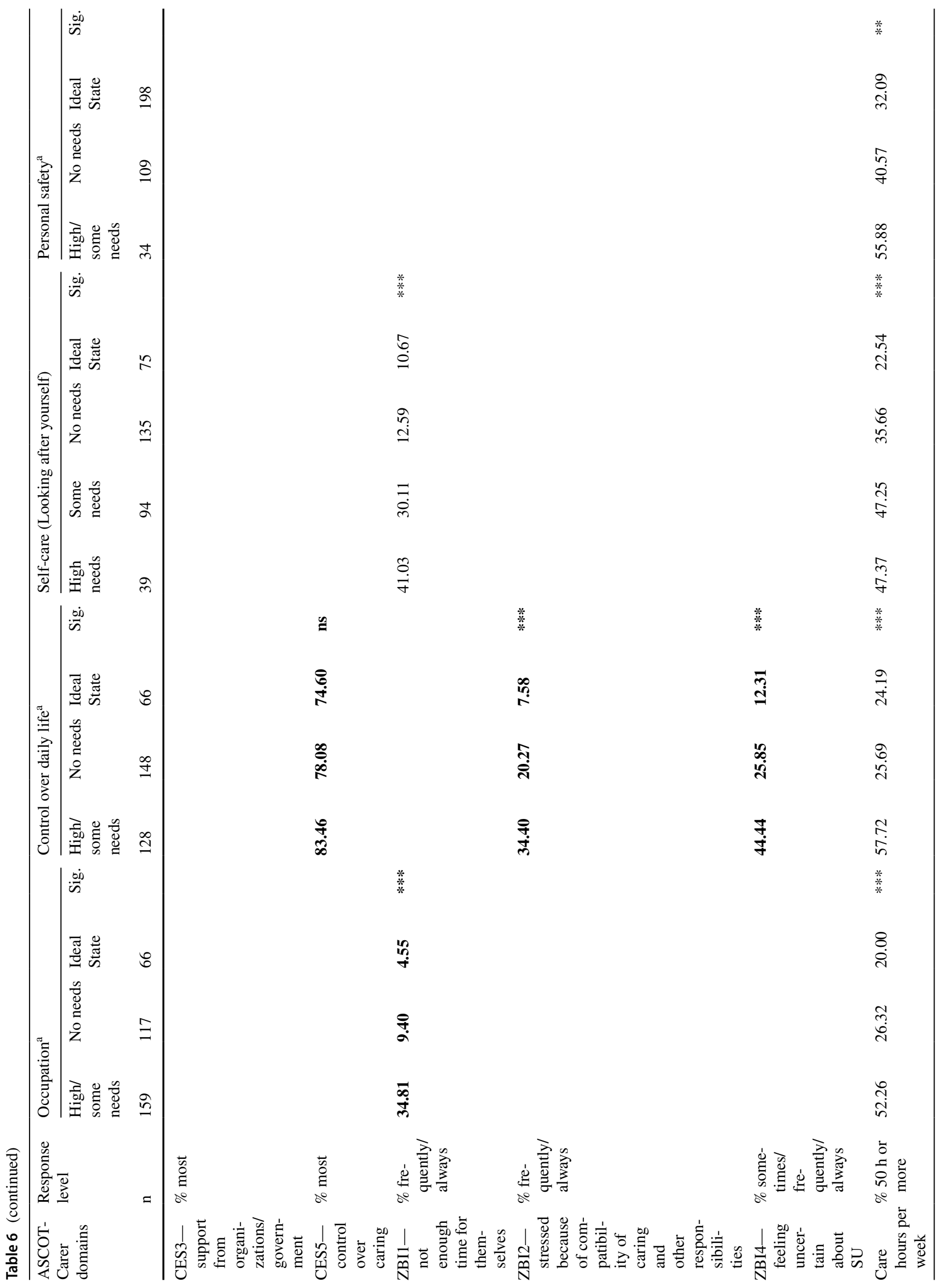




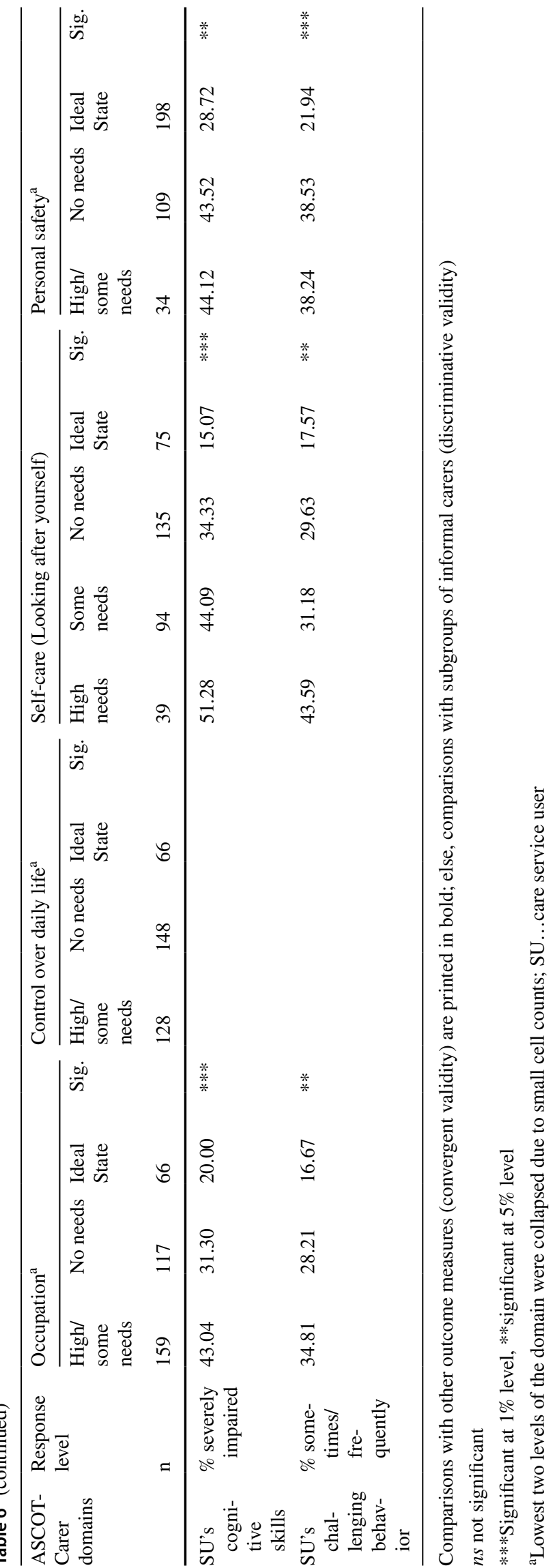

\section{Self-care (Looking after yourself)}

As hypothesized, the EQ-5D item 'self-care' (h-care1) was significantly positively at the $5 \%$ level, and ZBI 'time for oneself' (h-care2) negatively at the $1 \%$ level related to the ASCOT-Carer Self-care (Looking after yourself) domain. The ASCOT-Carer item Self-care was associated negatively with both the service user's cognitive performance (h-care4) and challenging behavior (h-care5) and care hours (h-care3), as expected (Tables 6 and 7).

\section{Personal safety}

All five EQ-5D items were significantly positively associated with the Personal safety domain (h-safe1). Also, in line with the hypotheses, we found significant negative associations between the ASCOT-Carer item and both service user cognitive skills (h-safe3), challenging behavior of the caredfor person (h-safe4) and care hours (h-safe2). As expected, informal carers with no problems in any of the EQ-5D-items, those whose cared-for person had no cognitive impairments or showed no challenging behavior, as well as informal carers who helped less than 50 hours a week, reported better Personal safety (Tables 6 and 7).

\section{Social participation and involvement}

Tables 6 and 7 show that, as expected, the ZBI item 'stressed because of compatibility of caring and other responsibilities' (h-soci1) as well as the number of weekly care hours (h-soci2) were each negatively related to the ASCOT-Carer Social participation and involvement domain. In line with the hypothesis, the social contact variables were also found to be positively related at the $1 \%$ level ('speak to neighbors', 'meet up with relatives/friends') and 5\% level ('speak to relatives/ friends) (h-soci3).

\section{Space and time to be yourself}

As expected, we found a significant positive association between the CES item 'activities outside caring' (h-time1) and the ASCOT-Carer item Space and time to be yourself. Negative associations were found between each of the variables (the ZBI item 'time for oneself' (h-time2) and care hours (h-time3)), while the possibility of taking breaks from caring and the ASCOT-Carer domain Space and time to be yourself were positively related (h-time4).

\section{Feeling supported and encouraged}

The ASCOT-Carer domain Feeling supported and encouraged was positively associated with the CES items 'support from family/friends' (h-supp1) and 'support from formal services' 


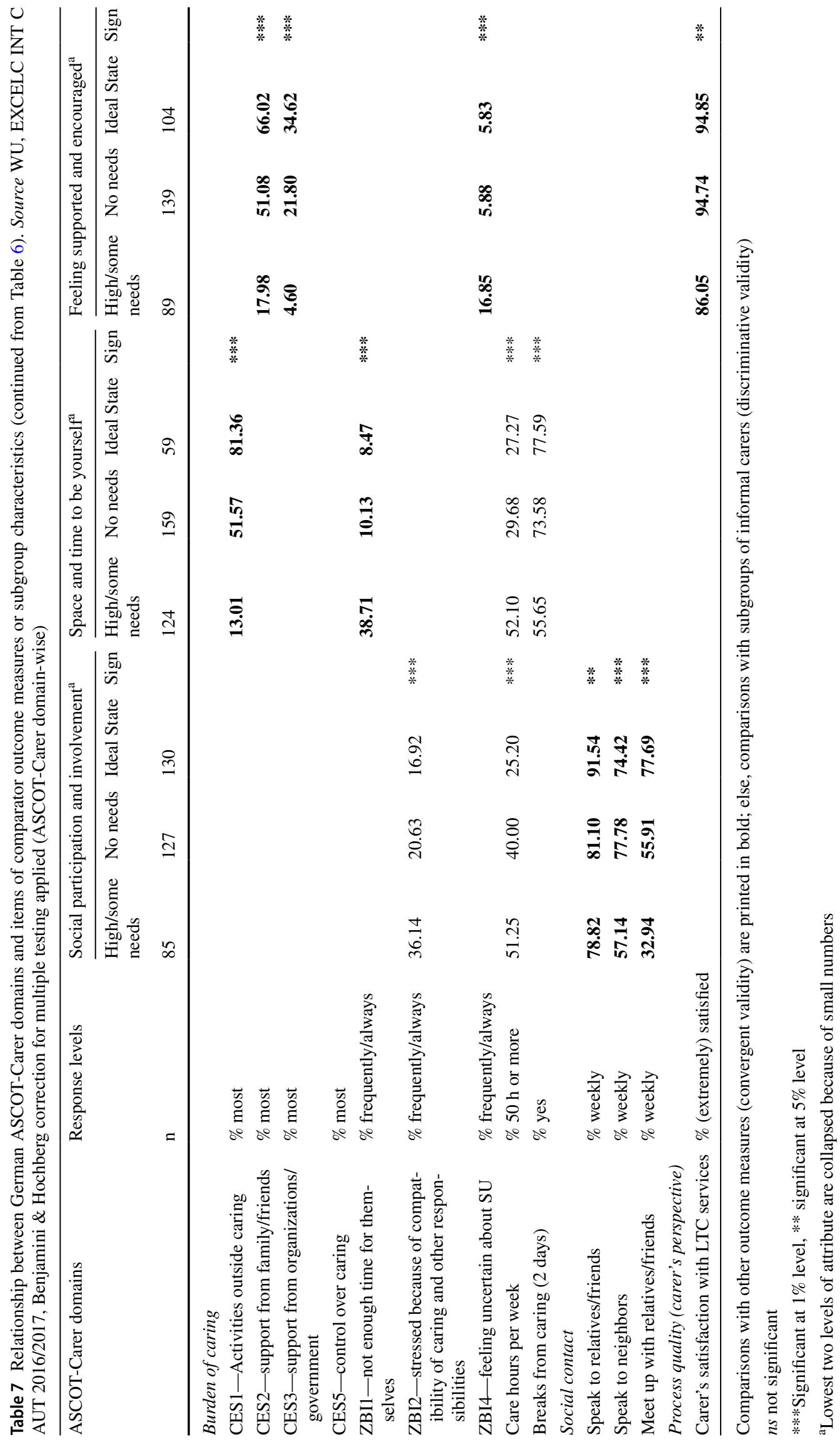


(h-supp2). We found the ZBI item 'feeling uncertain about the person cared for' (h-supp3) to be negatively related (significant at the 1\% level) with the ASCOT-Carer domain Feeling supported and encouraged. Finally, the process quality variable (overall satisfaction with LTC services) was also significantly associated with ASCOT-Carer Feeling supported and encouraged (see Tables 6 and 7).

\section{Discussion}

The translation and cultural adaptation according to ISPOR TCA guidelines aimed to develop a German version of the ASCOT-Carer instruments (INT4, SCT4) that has sufficient linguistic, content and construct validity and can be employed in German-speaking surveys as a measure of LTCQoL of informal carers.

The analysis of cognitive debriefing interviews provided evidence for linguistic and content validity. The interviews showed no major issues with comprehension of the German translation of ASCOT-Carer, except for the Personal safety domain, where the restriction to 'as a result of caring role' was not always picked up by the informal carers. Therefore, to ensure the understanding of the Personal safety domain, we recommend briefing interviewers to pay extra attention when addressing the domain and to stress the importance of the prompt included in the question.

In addition, we found solid evidence to support convergent validity as part of construct validity of the translated German ASCOT-Carer. The ASCOT-Carer score was significantly correlated with other measures of conceptuallyrelated constructs, particularly to scales also capturing carers' quality of life (such as EQ-5D index and measures of carers' experience and burden). As to be expected, the weakest association was found with the EQ-5D index that seeks to capture health-care instead of long-term care-related QoL.

For most of the ASCOT-Carer domains, significant associations with conceptually related constructs were found, except for Personal Safety, with no corresponding alternate measure in the data, and for the Control over daily life domain and the CES item 'control over caring', which seem to measure different aspects of having control. The ASCOTCarer domain Control over daily life was intended to reflect a broader concept that may also be influenced by other areas of life, not only caring [34], while the CES item has a narrower, more specific focus.

The comparison between subgroups of informal carers to explore discriminative or known-group validity focused on characteristics that are well supported by previous studies. As expected, informal carers with high care intensity or no opportunity to take a break from caring showed lower LTCQoL, as informal carers who cared for service users with low cognitive skills and challenging behavior.
While the analyses presented in this paper supported the construct validity of a culturally adapted German version of ASCOT-Carer and may be useful as a reference for assessing the validity of ASCOT-Carer translated to other languages, there are some limitations to this study. To begin with, we did not match English and Austrian samples of informal carers to investigate cross-cultural validity as defined by COSMIN study design checklist [13]. Second, we did not investigate measurement properties that require standardized interview data at two time points (e.g. test-retest reliability and responsiveness, such as the sensitivity of the instrument to changes of LTC service receipt over time) as this was beyond the financial means of this project. We thus encourage future research to assess these measurement properties.

The findings of this study provided good evidence for a culturally adapted German version of the ASCOT-Carer instrument. The cognitive debriefing interviews support its linguistic and content validity. Since almost all related constructs were significantly associated with the German ASCOT-Carer (score and individual domains) and in the expected direction (convergent validity), and since the same holds true sub-groups of informal carers (discriminative or known-group validity), there is good evidence for its construct validity. Furthermore, the analysis of qualitative as well as quantitative data comes to similar conclusions as reported for the original English ASCOT-Carer instrument $[2,3]$ and therefore support the construct validity of the German translation. These findings support the use of the German ASCOT-Carer instrument to capture LTC-related QoL for informal carers in Austria and other German speaking countries and can thus be utilized for national evaluations of LTC outcomes and comparative studies. Research is encouraged to assess further measurement properties of the translated instrument.

Acknowledgements We would like to thank the English ASCOT development team at PSSRU (University of Kent) for their valuable advice and support during the translation process. We thank Judith Kieninger (WU Research Institute for Economics of Aging) for her assistance with qualitative data collection and analysis and Tobias Krüse (WU Research Institute for Economics of Aging, until September 2016) and Meika Sternkopf (WU Research Institute for Economics of Aging, until July 2016) for their contributions to the translation process. Data collection was supported by the Austrian Federal Ministry of Labor, Social Affairs, Health and Consumer Protection, by long-term care organizations and local authorities in Austria.

Funding Open access funding provided by Austrian Science Fund (FWF). This project was funded by the NORFACE Welfare State Futures programme under grant number 462-14-160. In addition, the Austrian contribution to this project was co-funded by the Austrian Science Fund (FWF, Project Number I 2252-G16) and the Vienna Social Fund (FSW). The views expressed are not necessarily those of the funders. 


\section{Compliance with ethical standards}

Conflict of interest The authors declare that they have no conflicts of interest.

Ethical approval The responsible university office at the WU Vienna University of Economics and Business (Vice-Rector for Research) has determined that no ethics clearance was needed under university regulations.

Informed consent All participants have provided written consent to participate in the study.

Open Access This article is licensed under a Creative Commons Attribution 4.0 International License, which permits use, sharing, adaptation, distribution and reproduction in any medium or format, as long as you give appropriate credit to the original author(s) and the source, provide a link to the Creative Commons licence, and indicate if changes were made. The images or other third party material in this article are included in the article's Creative Commons licence, unless indicated otherwise in a credit line to the material. If material is not included in the article's Creative Commons licence and your intended use is not permitted by statutory regulation or exceeds the permitted use, you will need to obtain permission directly from the copyright holder. To view a copy of this licence, visit http://creativecommons.org/licenses/by/4.0/.

\section{References}

1. Colombo, F., Llena-Nozal, A., Mercier, J., \& Tjadens, F. (2011). Help wanted? Providing and paying for long-term care. OECD Health Policy Studies, Paris: OECD Publishing.

2. Rand, S. E., Malley, J. N., \& Netten, A. P. (2012). Measuring the social care outcomes of informal carers. An interim technical report for the identifying the impact of social care (IIASC) study, PSSRU Discussion Paper 2833. Personal Social Services Research Unit.

3. Rand, S. E., Malley, J. N., Netten, A. P., \& Forder, J. E. (2015). Factor structure and construct validity of the Adult Social Care Outcomes Toolkit for Carers (ASCOT-Carer). Quality of Life Research, 24(11), 2601-2614.

4. Al-Janabi, H., Flynn, T. N., \& Coast, J. (2011). Estimation of a Preference-Based Carer Experience Scale. Medical Decision Making, 31(3), 458-468.

5. Goranitis, I., Coast, J., \& Al-Janabi, H. (2014). An investigation into the construct validity of the Carer Experience Scale (CES). Quality of Life Research, 23(6), 1743-1752.

6. Joseph, S., Becker, S., Elwick, H., \& Silburn, R. (2012). Adult carers quality of life questionnaire (AC-QoL): development of an evidence-based tool. Mental Health Review Journal, 17(2), 57-69.

7. Zarit, S. H., Reever, K. E., \& Bach-Peterson, J. (1980). Relatives of the impaired elderly: correlates of feelings of burden. Gerontologist, 20, 649-655.

8. Zarit, S. H., \& Zarit, J. M. (1987). Instructions for the burden interview. University Park: Pennsylvania State University.

9. Netten, A., Burge, P., Malley, J., Potoglou, D., Towers, A-M., Brazier, J., et al. (2012). Outcomes of social care for adults: developing a preference-weighted measure. Health Technology Assessment, 16(16), 1-166.

10. Willis, G. B. (2015). The practice of cross-cultural cognitive interviewing. Public Opinion Quarterly, 79(S1), 359-395.
11. Beaton, D. E., Bombardier, C., Guillemin, F., \& Ferraz, M. B. (2000). Guidelines for the process of cross-cultural adaptation of self-report measures. Spine, 25(24), 3186-3191.

12. Wild, D., Grove, A., Martin, M., Eremenco, S., McElroy, S., Verjee-Lorenz, A., \& Erikson, P. (2005). Principles of good practice for the translation and cultural adaptation process for patientreported outcomes (PRO) measures: report of the ISPOR task force for translation and cultural adaptation. Value in Health, $8(2)$, 94-104.

13. Mokkink, L. B., Prinsen, C. A., Patrick, D. L., Alonso, J., Bouter, L. M., De Vet, H., et al. (2019). COSMIN Study Design checklist for Patient-reported outcome measurement instruments. Version July 2019, Retrieved May 18, 2020, from https://www.cosmin.nl/ wp-content/uploads/COSMIN-study-designing-checklist_final .pdf.

14. Avila, M. L., Stinson, J., Kiss, A., Brandão, L. R., Uleryk, E., \& Feldman, B. M. (2015). A critical review of scoring options for clinical measurement tools. BMC Research Notes, 8, 612 (2015).

15. Coltman, T., Devinney, T. M., Midgley, D. F., \& Venaik, S. (2008). Formative versus reflective measurement models: Two applications of formative measurement. Journal of Business Research, 61(12), 1250-1262.

16. Kimberlin, C. L., \& Winterstein, A. G. (2008). Validity and reliability of measurement instruments used in research. American Journal of Health-System Pharmacy, 65(23), 2276-2284.

17. PharmaQuest Ltd. (2016). Translation and Linguistic Validation of the ASCOT INT4 Service Users, SCT4 Service Users, INT4 Carers and SCT4 Carers into Finnish (Finland) and German (Austria)-Final Report. Unpublished manuscript.

18. Willis, G. B. (2005). Cognitive interviewing: A tool for improving questionnaire design. Thousand oaks: Sage Publications Inc.

19. Trukeschitz, B., Litschauer, J., Kieninger, J., Schoch, A., \& Hajji, A. (2018). EXCELC-EXploring Comparative Effectiveness and efficiency in Long-term Care: Study design and descriptive statistics for the Austrian sample of informal carers: Discussion Paper of the EXCELC Project No 3/2018 and Discussion Paper No 5/2018 of the WU Research Institute for Economics of Aging, Vienna University of Economics and Business, Austria.

20. Eremenco, S. L., Cella, D., \& Arnold, B. J. (2005). A comprehensive method for the translation and cross-cultural validation of health status questionnaires. Evaluation \& the Health Professions, $28(2), 212-232$

21. Knafl, K., Deatrick, J., Gallo, A., Holcombe, G., Bakitas, M., Dixon, J., \& Grey, M. (2007). The analysis and interpretation of cognitive interviews for instrument development. Research in Nursing \& Health, 30(2), 224-234.

22. Mokkink, L. B., Terwee, C. B., Patrick, D. L., Alonso, J., Stratford, P. W., Knol, D. L., et al. (2010). The COSMIN study reached international consensus on taxonomy, terminology, and definitions of measurement properties for health-related patient-reported outcomes. Journal of Clinical Epidemiology, 63(7), 737-745.

23. Brod, M., Tesler, L. E., \& Christensen, T. L. (2009). Qualitative research and content validity: Developing best practices based on science and experience. Quality of Life Research, 18(9), 1263-1278.

24. Mokkink, L. B., De Vet, H. C., Prinsen, C. A., Patrick, D. L., Alonso, J., Bouter, L. M., \& Terwee, C. B. (2018). COSMIN Risk of Bias checklist for systematic reviews of Patient-Reported Outcome Measures. Quality of Life Research, 27(5), 1171-1179.

25. COSMIN Risk of Bias Checklist. Retrieved May 18, 2020, from https://www.cosmin.nl/wp-content/uploads/COSMIN-RoB-check list-V2-0-v17_rev3.pdf.

26. EuroQol Group. (1990). EuroQol—A new facility for the measurement of health-related quality of life. Health Policy, 16(3), 199-208. 
27. Claes, C., Greiner, W., Uber, A., \& Graf von der Schulenburg, M. (1999). An interview-based comparison of the TTO and VAS values given to EuroQol states of health by the general German population. Paper presented at the Proceedings of the 15th Plenary Meeting of the EuroQol Group. Centre for Health Economics and Health Systems Research, University of Hannover.

28. Bédard, M., Molloy, D. W., Squire, L., Dubois, S., Lever, J. A., \& O'Donnell, M. (2001). The Zarit Burden Interview: a new short version and screening version. The Gerontologist, 41(5), 652-657.

29. Cohen, J. (1988). Statistical power analysis for the social sciences (2nd ed.). Hillsdale, New Jersey: Lawrence Erlbaum Associates.

30. Benjamini, Y., \& Hochberg, Y. (1995). Controlling the false discovery rate: a practical and powerful approach to multiple testing. Journal of the Royal Statistical Society: Series B (Methodological), 75(1), 289-300.

31. Olejnik, S., \& Algina, J. (2003). Generalized eta and omega squared statistics: measures of effect size for some common research designs. Psychological Methods, 8(4), 434-447.
32. Abma, I. L., Rovers, M., \& van der Wees, P. J. (2016). Appraising convergent validity of patient-reported outcome measures in systematic reviews: Constructing hypotheses and interpreting outcomes. BMC Research Notes, 9(1), 226.

33. StataCorp. (2017). Stata statistical software: Release 15. College Station, TX: StataCorp LLC.

34. Martire, L. M., \& Stephens, M. A. P. (2003). Juggling parent care and employment responsibilities: The dilemmas of adult daughter caregivers in the workforce. Sex Roles, 48(3/4), 167-173.

Publisher's Note Springer Nature remains neutral with regard to jurisdictional claims in published maps and institutional affiliations. 